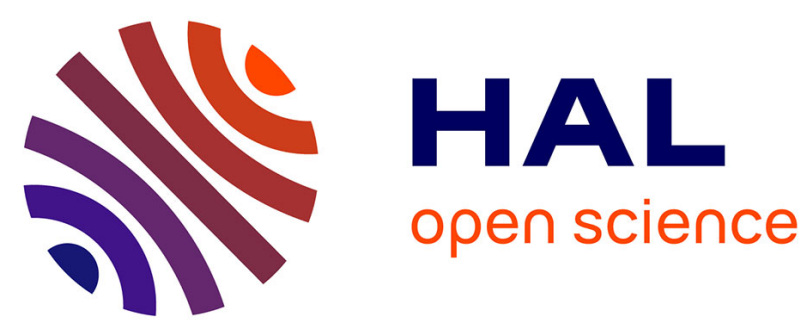

\title{
La spécificité de la recherche francophone en Systèmes d'Information
}

\author{
Sylvie Desq, Bernard Fallery, Robert Reix, Florence Rodhain
}

\section{To cite this version:}

Sylvie Desq, Bernard Fallery, Robert Reix, Florence Rodhain. La spécificité de la recherche francophone en Systèmes d'Information. Revue Française de Gestion, 2007, 33 (4), pp.63-80. 10.3166/RFG.176.63-79 . hal-00773737v2

\section{HAL Id: hal-00773737 \\ https://hal.science/hal-00773737v2}

Submitted on 12 Dec 2018

HAL is a multi-disciplinary open access archive for the deposit and dissemination of scientific research documents, whether they are published or not. The documents may come from teaching and research institutions in France or abroad, or from public or private research centers.
L'archive ouverte pluridisciplinaire HAL, est destinée au dépôt et à la diffusion de documents scientifiques de niveau recherche, publiés ou non, émanant des établissements d'enseignement et de recherche français ou étrangers, des laboratoires publics ou privés. 


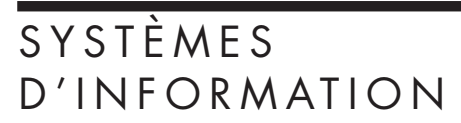

\section{La spécificité de la recherche francophone en systèmes d'information}

\author{
La recherche francophone \\ « suit-elle » la recherche \\ anglophone ou bien s'en \\ différencie-t-elle? \\ Concernant le champ des \\ systèmes d'information (SI) \\ auquel cet article est \\ consacré, les travaux \\ anglophones et \\ francophones s'intéressent- \\ ils aux mêmes \\ problématiques, aux mêmes \\ domaines d'application, \\ choisissent-ils les mêmes \\ niveaux d'analyse, se \\ réfèrent-ils aux mêmes \\ épistémologies, utilisent-ils \\ les mêmes méthodologies? \\ Après un rappel des \\ résultats obtenus sur \\ 25 ans de littérature en SI, \\ les auteurs présentent, en \\ comparant 763 articles de \\ recherche, 15 ans de \\ recherche anglophone et \\ francophone. Une troisième \\ partie présente les \\ évolutions historiques, en \\ séparant les deux \\ échantillons.
}

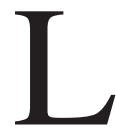

es systèmes d'information se sont constitués en champ de recherche il y a seulement 25 ans : aux États-Unis la revue MISQ (Management Information Systems Quaterly) a commencé à paraître en 1977, et ICIS (International Conference on Information Systems) a débuté en 1980. La revue francophone TIS (Technologie Information et Société) a été publiée jusqu'en 1996, en France les journées nationales des IAE proposent depuis 1984 une session pour la discipline, et depuis 1996 le colloque de l'AIM (Association information et management ; http://www.aim.asso.fr/) et la revue SIM (Systèmes d'information et management http://revuesim.free.fr) se sont finalement imposés comme les lieux de publication académique.

Depuis 1980 de nombreux auteurs ont cherché à classer les recherches en systèmes d'information. Au cours d'une recherche précédente, nous avions proposé un tableau pour comparer tous ces «états de l'art» en systèmes d'information (Desq et al., 2002). Une première partie rappellera donc ici comment on peut décrire le champ de recherche: la grille que nous avions utilisée,

\footnotetext{
* Robert Reix a été à l'origine de ce travail d'analyse. Il a été le directeur de thèse des trois autres auteurs et bien souvent leur « guide ». Il nous a quitté brutalement le 22 février 2006 et signe là, à titre posthume, cette publication à laquelle il a largement contribué.
} 
et les principaux résultats qui avaient été obtenus sur 25 ans de littérature de 1977 à 2001.

Mais cette représentation globale de la recherche en systèmes d'information ne masque-t-elle pas des différences culturelles fortes? Autrement dit, les chercheurs anglophones et francophones s'intéressentils aux mêmes problématiques, aux mêmes domaines d'application, observent-ils les mêmes niveaux d'analyse, se réfèrent-ils aux mêmes épistémologies, utilisent-ils les mêmes méthodologies? La deuxième partie présentera les résultats obtenus en comparant les recherches francophones et anglophones sur une période commune: 19872001.

Les résultats ainsi exposés ne tiennent pas compte de la dimension temporelle. Est-il possible de voir aussi, chez les anglophones et les francophones, une évolution différente des préoccupations ou des méthodes? En séparant maintenant les deux échantillons, et en les analysant par périodes de trois années, on constate effectivement des variations différentes dans les deux groupes. La troisième partie présentera ces évolutions historiques.

\section{I. - COMMENT DÉCRIRE LE CHAMP DES SYSTÈMES D'INFORMATION?}

\section{Une nouvelle vision du champ de recherche: une grille à sept dimensions}

Décrire un champ de recherche et son évolution pose de redoutables difficultés. De nombreux choix sont à faire: Quelle grille faut-il adopter pour qualifier les travaux retenus? Quelle base de références faut-il retenir? Quelle période faut-il étudier? À ces trois questions, les chercheurs ont apporté des réponses très différentes; nous avons nous-mêmes fait nos propres choix, qu'il semble important de justifier.

Il existe bien des façons d'analyser un champ de recherche. On peut s'intéresser aux références bibliographiques citées dans les articles, afin de voir si le champ émerge comme un domaine indépendant et s'il existe une tradition de recherche cumulative (Culnan et Swanson, 1986; Culnan, 1987; Cheon et al., 1991; Hamilton et Ives, 1982; Suomi, 1993). On peut aussi chercher à identifier les problèmes-clés des professionnels, afin d'orienter la recherche vers plus de pertinence (Dickson et al., 1984 ou Brancheau et al., 1996). Plus souvent, on cherche à construire des « grilles » permettant la classification de différentes productions du domaine (articles, communications, thèses). En systèmes d'information, Ives, Hamilton et Davis ont été les premiers à définir cinq types de recherche et à procéder à la classification d'un certain nombre de thèses et d'articles (Ives et al., 1980 ; Hamilton et Ives, 1982). Depuis, par des démarches de regroupement de motsclés, des grilles plus fines ont été développées (56 catégories par Barki et al., (1988, 1993), 37 catégories par Swanson et Ramiller 1993, 48 catégories par Lee et al., 1999)). Ces grilles ont été utilisées voire adaptées par différents chercheurs (Cheon et al., 1993; Alavi et Carlson, 1992; Reix et Fallery, 1996; Claver et al., 2000; Peaucelle, 2001).

Mais toutes les grilles proposées jusqu'ici n'étaient que des listes « unidimensionnelles». Elles n'ont donc pas pu être définies selon des critères homogènes: elles englobent aussi bien des sujets qui sont de 
véritables questions de recherche (comme l'implantation, l'évaluation des SI, etc.), que d'autres qui sont des outils (comme les systèmes d'aide à la décision, le travail coopératif assisté par ordinateur, etc.) ; elles mélangent le niveau d'analyse (individu, organisation, interorganisation) et l'objet d'analyse (l'information, la technologie, l'organisation). C'est pourquoi nous avions adopté en 2002 une démarche déductive pour définir une vision multidimensionnelle du champ de recherche avec sept grandes dimensions (voir Desq et al., 2002). Une recherche en SI peut alors se définir en premier lieu par sa problématique, se regroupant en trois grands thèmes : la gestion stratégique des SI, le développement des SI, et le contrôle des SI (le détail de ces problématiques est donné à la fin de l'article: 13 problématiques initiales, regroupées ensuite en 6 problématiques plus larges pour les traitements statistiques). Mais on peut ensuite distinguer cinq grands domaines d'application: l'informationnel (données et connaissances), le fonctionnel (transactions et opérations), le décisionnel (processus et aide à la décision), le relationnel (processus et aide à la communication) et un domaine général (le SI d'une organisation pris dans sa globalité). L'objet d'une recherche peut être classé comme conceptuel (l'information, la décision), technique (le fonctionnement d'un outil) ou organisationnel (l'intersection de la technique et de l'organisation). La perspective peut être l'ingénierie (ex-ante) ou l'intégration des systèmes d'information dans les organisations (ex-post). Le niveau d'analyse peut être l'individu, le groupe, l'organisation, plusieurs organisations, ou la société. Les publications peuvent être théoriques ou empiriques, pour ces dernières l'épistémologie peut être positiviste ou interprétative et les méthodologies peuvent être diverses (enquêtes, études de cas, recherche action, expérimentation, etc.). Pour ce qui est du choix des sources bibliographiques, la méthodologie de comptage est sensible aux choix du corpus étudié, et on constate des positions différentes selon les études. Dans les premiers travaux descriptifs du champ, les écrits publiés dans des revues ou colloques étiquetés « systèmes d'information » (MISQ, JMIS, ISR, Info \& Mgt, ICIS) sont analysés au côté d'articles provenant de revues généralistes (AMJ, AMR, ASQ, SMR, HBR, Management Science). Deux raisons justifiaient alors ce choix: la littérature en systèmes d'information en était à ses débuts, et les analyses cherchaient justement à savoir si les systèmes d'information étaient en train d'émerger comme un champ théorique « autonome », par rapport aux sciences de l'informatique et du management. Le choix des revues consultées se justifie donc par les questions posées par chaque analyse. Ainsi, Lee et al. (1999), voulant comparer les thématiques des chercheurs et des praticiens en systèmes d'information, ont étudié des publications de revues de recherche et de magazines professionnels. Swanson et Ramiller (1993) ont à l'inverse restreint leur analyse à une seule référence, puisque leur objectif était de connaître l'image du domaine à travers une nouvelle revue (56 articles acceptés sur un total de 395 propositions, soit $14 \%$, mais avec une variation des taux d'acceptation qui indique une politique éditoriale: en 5 ans le taux avait par exemple baissé de $40 \%$ à $4 \%$ sur les thèmes «projets, évaluation et stratégie »). Grover et al. (1993) sélectionnent les articles dans les revues spécialisées $(80 \%)$ 
et dans les revues généralistes $(20 \%)$ pour s'interroger sur le degré de rigueur des articles publiés, en jugeant si l'article satisfait aux critères classiques: constitution de l'échantillon, représentativité, méthode de collecte, méthode d'analyse statistique (leur score varie de 0 à 9 , il est en moyenne légèrement supérieur à 3 , et la rigueur de ces études empiriques par enquête ne semble pas s'être améliorée). Reix et Fallery (1996) ont analysé 510 publications en repérant trois étapes de production scientifique (la thèse, la communication au congrès ICIS et la publication dans la revue MISQ) : les thèses sur l'évaluation auraient beaucoup plus de chance de donner lieu à des articles (84\% d'acceptation) que les thèses sur l'introduction des systèmes d'information ou sur les SIAD (25\% d'acceptation). Peaucelle (2001) s'est intéressé aux 104 auteurs d'articles de SIM publiés en 5 ans, et montre que les chercheurs français publient souvent seuls (59 \%, à l'inverse des auteurs américains, $17 \%$ des articles de MISQ, et des britanniques où ce taux est tombé de $42 \%$ à $26 \%$ dans la revue ISJ), et qu'un noyau dur de 17 auteurs prolifiques assure un rôle central dans le domaine (comité de lecture, association AIM, articles publiés).

Pour nous, qui voulions comprendre l'évolution du champ de recherche et faire des comparaisons internationales, la principale revue de recherche (MIS Quaterly) et les actes de la plus grande conférence étaient incontournables (ICIS, dont les travaux ont été plus rarement analysés). Les précédents états de l'art ne se sont appuyés que sur cette littérature anglophone, car c'est bien sûr la principale littérature existante dans le domaine. Mais c'est aussi de notre responsabilité, chercheurs francophones en sys- tèmes d'information, de nous interroger sur « notre » production. Ce que nous appelons ici la recherche francophone est l'ensemble des publications et communications dans les revues et les colloques de langue française (TIS et SIM pour les revues, IAE et AIM pour les congrès).

En ce qui concerne la période étudiée, nous avions voulu qu'elle soit suffisamment importante pour véritablement percevoir des évolutions, alors que peu de travaux de description du champ des systèmes d'information avaient retenu une période aussi longue.

\section{Le contenu de la recherche en systèmes}

d'information depuis 25 ans : l'importance de l'évaluation des nouvelles technologies

Cette nouvelle grille de lecture à sept dimensions a d'abord été appliquée à 25 ans de littérature (1977-2001), et 1018 articles avaient été analysés dans leur intégralité (non pas seulement la simple lecture des résumés ou des mots-clés, mais bien les articles eux-mêmes). Pour limiter la subjectivité du classement il a été vérifié sur un échantillon préalable que les quatre lecteurs convergeaient dans leurs analyses (Desq et al., 2002). On peut rappeler ici les résultats obtenus dans cette étude globale.

Dans la production scientifique internationale le thème général du contrôle (animation, évaluation et gestion des personnels informaticiens) dominait la discipline avec $45 \%$ des articles, loin devant le développement $(28 \%)$ et la stratégie (23\%). L'objet de la discipline, comme le niveau d'analyse, était bien l'organisation (84\%), l'épistémologie positiviste était dominante (45\%), les domaines décision et communication l'emportaient sur l'informationnel et le fonctionnel. La perspective « ex-post» 
dominante ne paraissait pas imposer un type méthodologique particulier.

Une analyse plus fine montrait enfin des liens significatifs entre les problématiques et les autres dimensions :

- la « gestion stratégique » des SI était liée au niveau d'analyse organisationnel et aux méthodes par entretiens,

- la « conception des SI » était liée aux perspectives d'ingénierie ex-ante et à une épistémologie de type théorique/normatif,

- la problématique de la « gestion de projets SI » était liée à un objet de recherche technique et à une épistémologie interprétative,

- enfin et surtout la problématique dominante de "l'évaluation des SI » était liée aux méthodologies expérimentales et à une épistémologie positiviste.

Dans cette même étude globale on constatait aussi plusieurs évolutions historiques: le développement (conception et gestion de projets SI) a perdu aujourd'hui son caractère majoritaire (avec l'émergence de la problématique de l'animation). Les perspectives « ex-ante » (très liées à l'ingénierie des systèmes d'information au départ de la discipline), sont aujourd'hui devenues minoritaires. À partir de 1985 le niveau d'analyse individuel, qui était dominant, a baissé au profit du niveau organisationnel devenu majoritaire, et du niveau interorganisationnel aujourd'hui émergent. De grandes variations historiques apparaissaient dans le recours à l'expérimental (sans que l'on puisse trouver d'explications très évidentes). Après un engouement éphémère pour les travaux techniques entre 1985 et 1988, on constatait un désintérêt accru pour ce type d'études aujourd'hui (mais elles n'ont jamais représenté que $7 \%$ des articles de l'échantillon). Un renversement s'est fait depuis 1995: le domaine du décisionnel, bien qu'encore majoritaire, a perdu de l'importance face au domaine informationnel (intérêt actuel pour la gestion des connaissances?). Enfin la perspective positiviste, qui est toujours aussi largement dominante dans notre champ, n'est plus seule (une épistémologie constructiviste minoritaire est aujourd'hui émergente, qui explique sans doute le recours récent aux recherchesactions).

\section{II. - QUINZE ANS DE RECHERCHES ANGLOPHONES ET FRANCOPHONES : DES INTÉRÊTS ET DES MÉTHODES DIFFÉRENTES}

\section{La constitution des deux échantillons : « travaux anglophones » et « travaux francophones »}

Ce que nous appelons ici la recherche francophone est donc l'ensemble des publications et communications dans les revues et les colloques de langue française (TIS, SIM, IAE, AIM). La recherche anglophone se compose quant à elle des articles parus dans la revue MISQ et des communications des différents congrès ICIS. Dans MISQ et ICIS les auteurs sont anglophones, à quelques rares exceptions. De la même manière les auteurs dans les congrès des IAE et de l'AIM ainsi que dans TIS et SIM sont très majoritairement des francophones (les Canadiens publiant dans TIS sont des Québécois qui rédigent en langue française).

Mais, pour comparer la littérature anglophone et francophone, nous ne pouvions pas disposer d'une période de 25 ans (19772001), puisque les données francophones ne sont repérables qu'à partir de 1987. En prenant alors 1987 comme année de départ, 
nous obtenons une base de comparaison homogène sur 15 ans, composée cette fois de 763 articles, dont $54 \%$ sont anglophones et $46 \%$ francophones (tableau 1).

Pour chacun des articles étudiés nous avons relevé le pays de l'institution à laquelle se rattache l'auteur principal. Cela nous permet de décrire plus en détail « qui se cache » derrière ces recherches dites francophones et anglophones (tableau 2). Il est important de noter alors que la partie anglophone représente très majoritairement l'Amérique du Nord (77\% des auteurs viennent des États-Unis, $6 \%$ du Canada, $4 \%$ de la GrandeBretagne). Il s'agit donc ici de prendre le mot anglophone dans une acception particulière. Quand à la partie francophone de l'échantillon, elle est constituée d'auteurs français pour $67 \%$, d'auteurs canadiens (il faut entendre là québécois) pour $20 \%$, et d'auteurs de pays non francophones pour $10 \%$. En regroupant en retenant uniquement le continent d'origine de l'auteur, on constate que notre échantillon de recherches anglophones regroupe pour l'essentiel des auteurs d'Amérique du Nord et que l'échantillon de recherches francophones se compose au trois quart de travaux mené par des Européens.

\begin{tabular}{|l|c|c|}
\hline \multicolumn{3}{|c|}{ Tableau 1 } \\
\hline \multicolumn{2}{|c|}{ REVUES ET COLLOQUES DANS L'ÉCHANTILLON SUR 15 ANS (1987-2001) } \\
\hline AIM & Nombre de citations & Part dans l'échantillon \\
\hline IAE & 71 & $9 \%$ \\
\hline SIM & 51 & $7 \%$ \\
\hline TIS & 100 & $13 \%$ \\
\hline Total des travaux francophones & 129 & $17 \%$ \\
\hline MISQ & $\mathbf{3 5 1}$ & $\mathbf{4 6} \%$ \\
\hline ICIS & 322 & $42 \%$ \\
\hline Total des travaux anglophones & 90 & $13 \%$ \\
\hline TOTAL & $\mathbf{4 1 2}$ & $\mathbf{5 4} \%$ \\
\hline
\end{tabular}

Tableau 2

ORIGINE DES AUTEURS DANS L'ÉCHANTILLON SUR 15 ANS (1987-2001)

\begin{tabular}{|l|c|c|c|c|}
\hline & \multicolumn{2}{|c|}{ Francophones } & \multicolumn{2}{c|}{ Anglophones } \\
\hline Amérique du Nord & 76 & $21 \%$ & 345 & $84 \%$ \\
\hline Europe & 254 & $72 \%$ & 17 & $4 \%$ \\
\hline Autres & 21 & $6 \%$ & 50 & $12 \%$ \\
\hline
\end{tabular}


2. Les recherches anglophones axées sur le développement de SI/francophones axées sur l'animation des SI

Il ressort de nos analyses statistiques sur 15 ans que les différences entre les recherches anglophones et francophones sont fortement significatives sur toutes les sept dimensions retenues. Tous les Chi2 sont significatifs; on donne dans le tableau 3 en exemple les croisements pour les problématiques.

\begin{tabular}{|l|c|c|c|c|c|c|c|}
\hline \multicolumn{7}{|c|}{$\begin{array}{c}\text { Tableau 3 } \\
\text { PROBLÉMATIQUES }\end{array}$} \\
\hline $\begin{array}{l}\text { Rroblématique } \\
\text { Revues }\end{array}$ & $\begin{array}{c}\text { Gestion } \\
\text { stratégique }\end{array}$ & Divers & Conception & $\begin{array}{c}\text { Gestion } \\
\text { de projet }\end{array}$ & Animation & Évaluation & Total \\
\hline Francophones & -8 & +10 & +0 & -4 & +24 & -8 & 351 \\
\hline Anglophones & +7 & -9 & +0 & +3 & -20 & +6 & 412 \\
\hline Total & $\mathbf{1 4 1}$ & $\mathbf{1 1 7}$ & $\mathbf{7 4}$ & $\mathbf{9 4}$ & $\mathbf{1 3 6}$ & $\mathbf{2 0 1}$ & $\mathbf{7 6 3}$ \\
\hline
\end{tabular}

Note : Les valeurs du tableau sont les pourcentages Chi2 partiel/Chi2 total, le signe représente l'écart à l'indépendance, et les chiffres en italique soulignent les contributions les plus fortes à l'indépendance des deux variables.

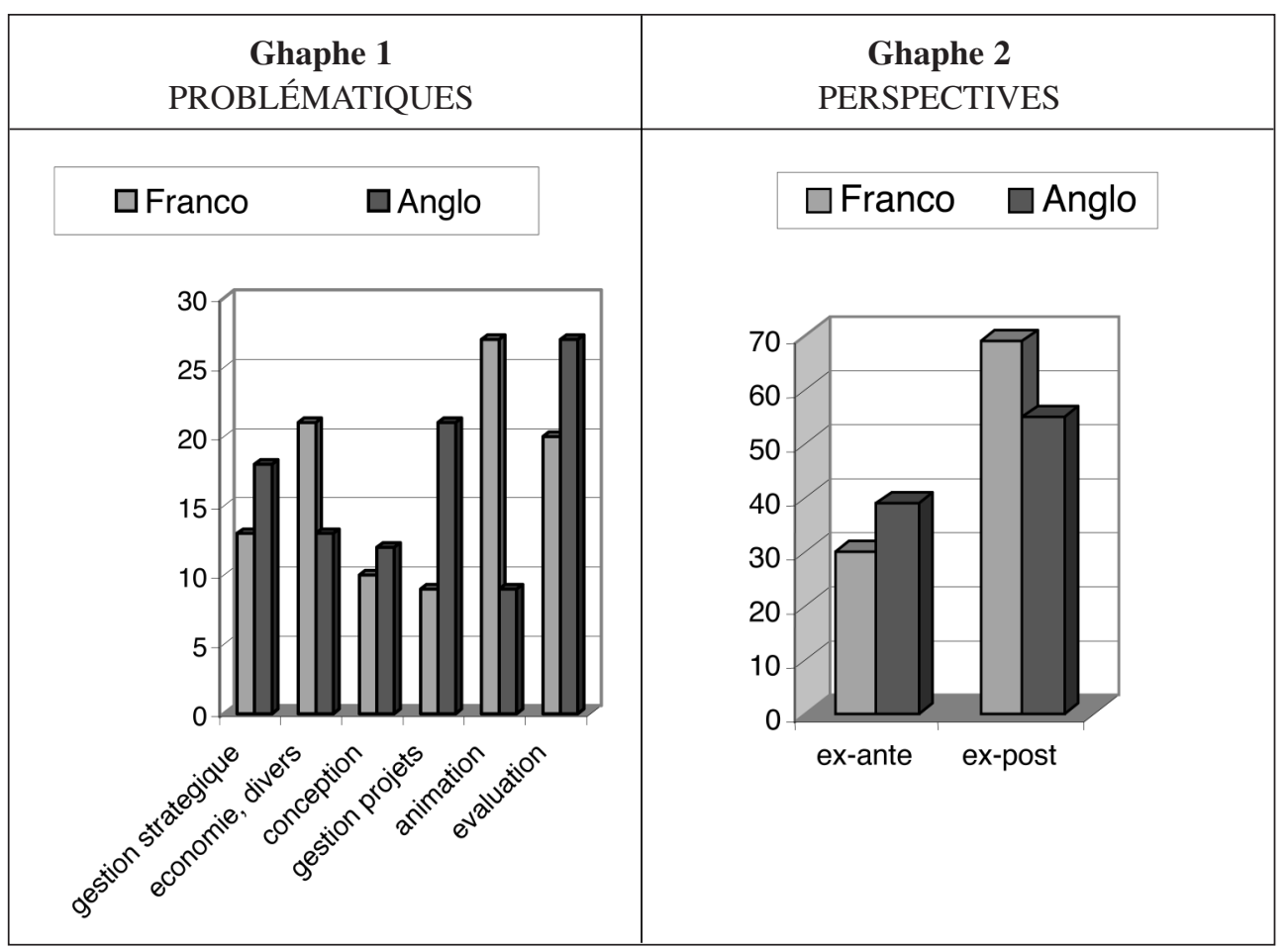


On constate tout d'abord que les recherches francophones concernent les problématiques d'animation, bien plus que les anglophones (graphe 1). Nous regroupons sous ce vocable animation: les études sur la conduite du changement organisationnel, l'appropriation des nouvelles technologies, la transformation des processus...

Les recherches anglophones, à l'inverse, ont conservé une importante préoccupation pour le développement, surtout dans sa composante gestion de projets: réutilisation, prototypage, évaluation des risques, méthodes de gestion de projet...

Ce résultat est à rattacher aux différences observées dans la perspective de la recherche (graphe 2) :

- les recherches anglophones n'ont pas délaissé la perspective d'ingénierie exante (certes minoritaire, $40 \%$ ),

- tandis que les recherches francophones se placent complètement dans une perspective d'intégration ex-post, $70 \%$ (bien naturellement liée aux problématiques d'animation).

En ce qui concerne l'objet de la recherche (graphe 3), les travaux francophones et anglophones se rapprochent par le centrage sur un objet d'étude: l'organisation (84\% !).

Malgré tout, le croisement est significatif (test du Chi2 tableau 4): les recherches anglophones ont une vision plus technique, alors que les recherches francophones sont plus souvent à orientation conceptuelle.

$\mathrm{Au} \mathrm{vu}$ de ces premières différences, on pourrait avancer l'idée que les travaux francophones cherchent davantage à comprendre des phénomènes, alors que les anglophones sont plus proactifs dans la création de méthodes et d'outils.
Les recherches anglophones et francophones n'adoptent pas non plus exactement les mêmes niveaux d'analyse. Même si le niveau d'analyse de notre discipline est très largement l'organisation, les travaux francophones ont une tendance plus marquée à se porter au niveau sociétal (13\% contre $1 \%$ pour les anglophones, des chiffres qui peuvent s'expliquer par la politique éditoriale de la revue francophone TIS jusqu'en 1996) ou interorganisationnel (10\% contre $2 \%$, notamment sur les réseaux d'organisations), et les travaux anglophones choisissent plus souvent le niveau du groupe. Parmi les cinq grands domaines d'application que nous avons distingués les recherches francophones portent plus sur un domaine précis: l'information (15\% contre $5 \%$ chez les anglophones) ou la communication ( $21 \%$ contre $7 \%$ ). Les travaux anglophones concernent plus le domaine global du SI

\section{Recherches anglophones positivistes expérimentales/francophones interprétativistes à base d'entretiens}

L'étude des épistémologies et des méthodologies montre là encore une différence notable entre les recherches francophones et anglophones.

Nous découvrons (ou nous confirmons) que les travaux francophones sont plus tournés vers les épistémologies de type interprétativiste $(30 \%$ contre $5 \%$ ) (graphe 4$)$.

Il aurait été intéressant de posséder dans notre panel d'analyse des journaux académiques européens autres que les seuls français, comme par exemple les revues anglaises en SI, pour vérifier si l'opposition souvent relevée dans le discours des chercheurs nord-américains, à savoir que 


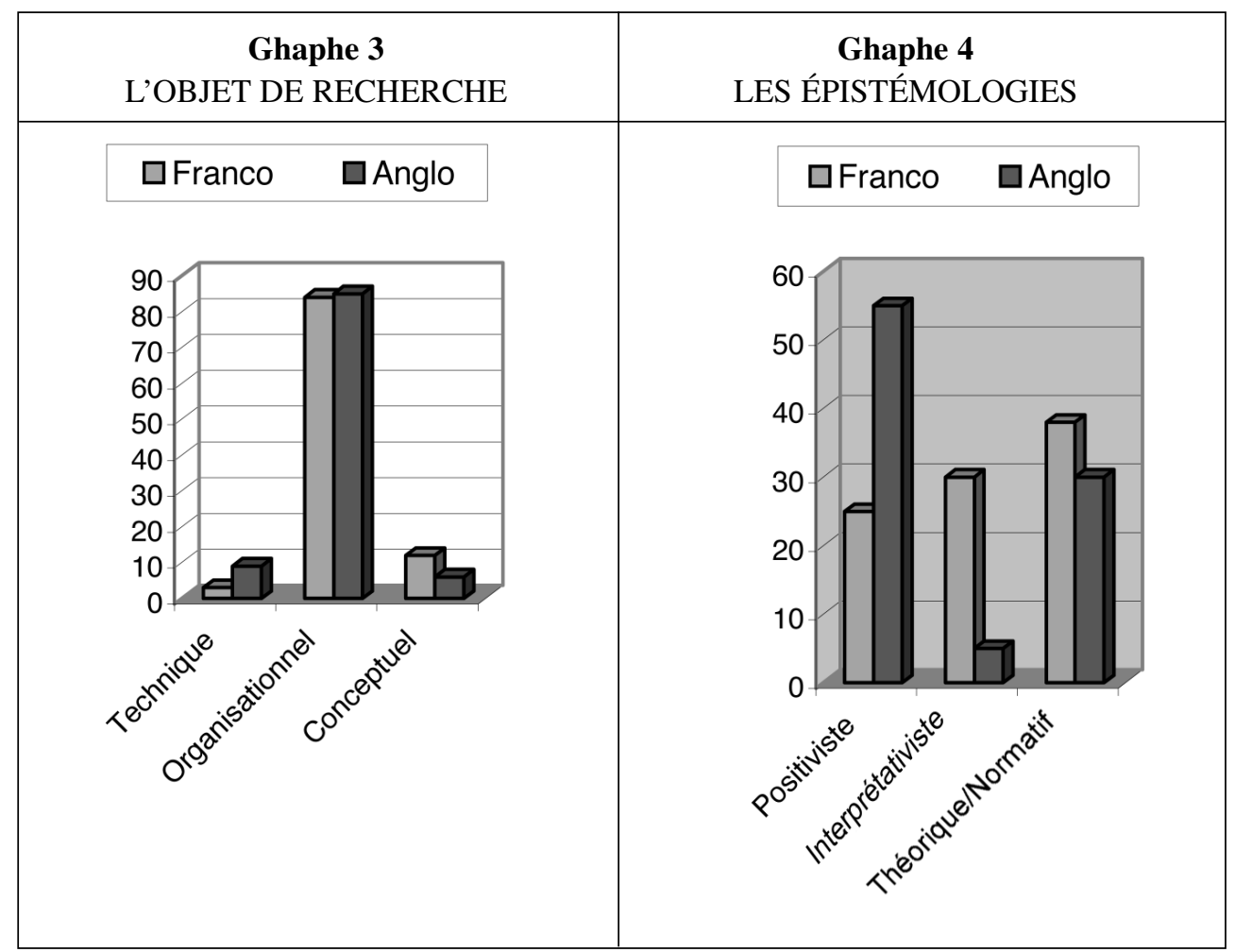

l'Europe est plus ouverte que l'Amérique $\mathrm{du}$ Nord aux épistémologies autres que positivistes, se confirme dans les statistiques.

Puisque épistémologie et méthodologie sont étroitement liées, on note logiquement une différence entre recherches francophones et anglophones dans le recours aux différentes méthodes de recherche (graphe 5). Ainsi :

- les travaux francophones utilisent moins souvent la technique des questionnaires, et

\begin{tabular}{|c|c|c|c|c|c|c|}
\hline \multicolumn{7}{|c|}{$\begin{array}{c}\text { Tableau } 4 \\
\text { MÉTHODES }\end{array}$} \\
\hline Revues & $\mathbf{Q}$ & EC & EX & ENT & RA & Total \\
\hline Francophones & -15 & +7 & -9 & +10 & +16 & 222 \\
\hline Anglophones & +11 & -5 & +7 & -8 & -12 & 302 \\
\hline Total & 179 & 183 & 79 & 66 & 17 & 524 \\
\hline
\end{tabular}

Méthodes : $\mathrm{Q}=$ questionnaires, $\mathrm{EC}=$ études de cas, $\mathrm{EX}=$ expérimentation, $\mathrm{ENT}=$ entretiens, $\mathrm{RA}=$ recherche action. Les valeurs du tableau sont les pourcentages Chi2 partiel/Chi2 total, le signe représente l'écart à l'indépendance, et les chiffres en italique soulignent les contributions les plus fortes à l'indépendance des deux variables. 


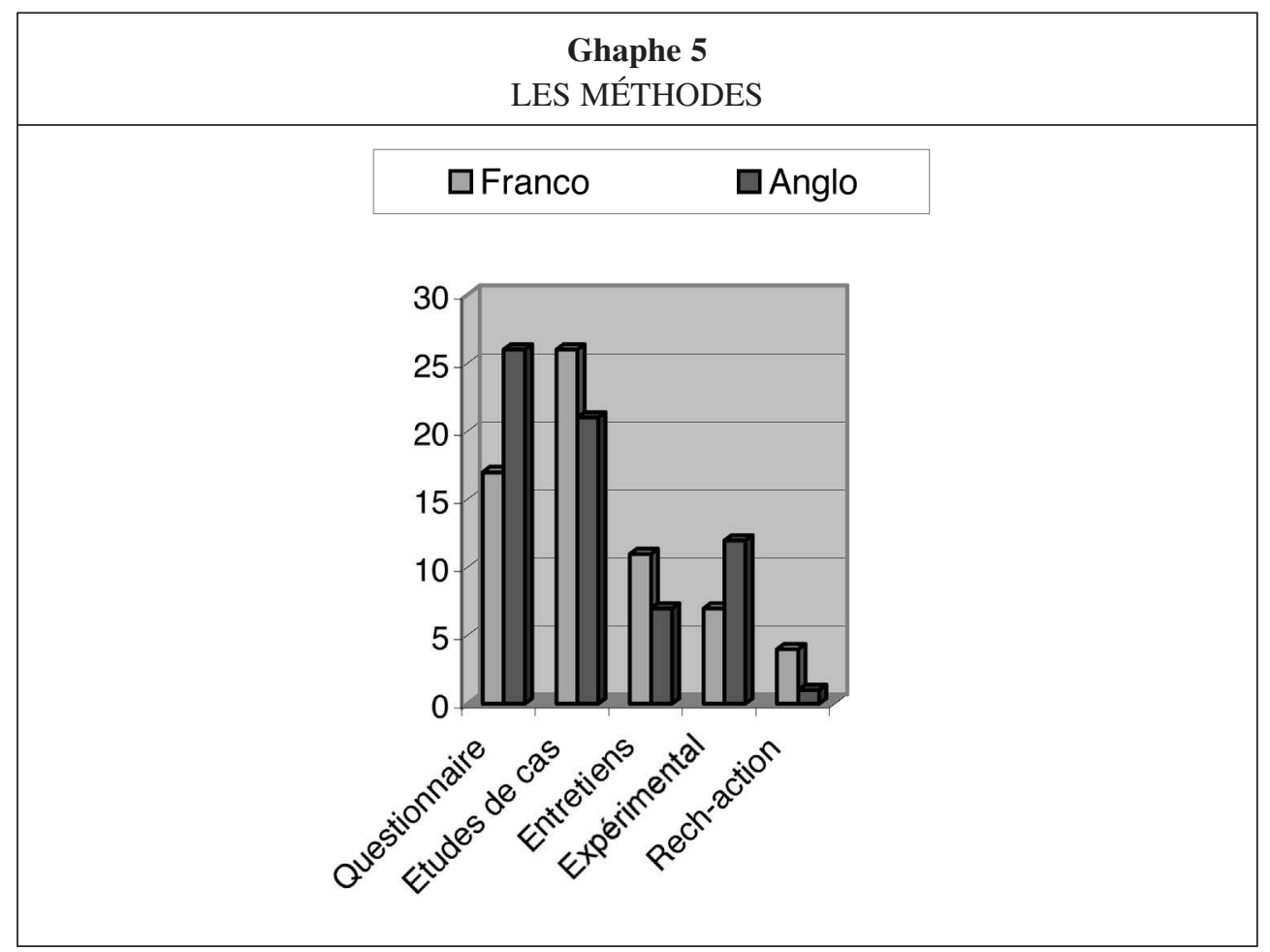

conduisent plus d'entretiens et de recherches-action que les anglophones,

- les publications anglophones ont conservé une tradition de méthodes expérimentales.

Notons également que dans la partie « anglophone » se retrouvent les articles parus dans ICIS et dans MISQ. Or, depuis ses origines, MISQ avait eu tendance à publier presque exclusivement des articles relevant d'une épistémologie positiviste. Mais depuis quelques années MISQ s'est ouvert aux autres épistémologies. Un signe fort a été donné par l'arrivée de Allen Lee à la tête de la rédaction de la revue. Dans le numéro de mars 1999, on peut lire dans le discours éditorial inaugural d'Allen Lee le commentaire suivant:
"Also, once seen as a bastion of quantitative and positivist research, MIS Quarterly now boasts excellent representation from qualitative, interpretive, and case researchers among its associate and senior editors (where the current editor-in-chief is a qualitative methodologist) and has even given its best paper award to qualitative articles. I will continue the progress in achieving diversity by seeking out and, as necessary, developing qualified scholars to join our Editorial Board."

Le ton est donné, et effectivement les numéros suivants de MISQ voient apparaître un nombre croissant d'articles se référant à d'autres épistémologies que la seule épistémologie positiviste. 
Comme l'explique Peaucelle (2001), le milieu académique régule la recherche en SI, et « le jugement des animateurs de la revue a une forte influence pour créer le mouvement des thèmes ». Au mouvement des thèmes s'ajoute à n'en pas douter celui des épistémologies et des méthodologies...

\section{III. - RECHERCHES ANGLOPHONES VERSUS FRANCOPHONES : DES ÉVOLUTIONS DIFFÉRENTES}

Suomi (1993) a bien montré, par une analyse comparative des références bibliographiques d'articles publiés dans six revues nord-américaines et dans six revues européennes, que les européens font surtout référence aux publications nord-américaines, alors qu'à l'inverse les travaux nord-américains citent très peu les européens.

La recherche anglophone en systèmes d'information ayant commencé 10 ans avant la recherche francophone, il paraît légitime de se demander si les travaux francophones se sont « inspirés » des écrits anglophones (« copier »?), et s'ils ont de façon générale adopté les mêmes préoccupations et les mêmes démarches.

\section{Les préoccupations des travaux anglophones et francophones: une autonomie de chaque communauté, mais pas de suivisme}

Nous avons calculé (successivement pour les recherches anglophones et francophones) les croisements entre les années et les sept dimensions de la grille: les problématiques, les perspectives, les niveaux d'analyse et les épistémologies. On constate alors une évolution différente dans les deux groupes.

En ce qui concerne les problématiques, les recherches anglophones précèdent les francophones dans l'intérêt porté à la gestion stratégique des systèmes d'information (dès 1990 pour les anglophones, pas avant 1996 pour les francophones). Mais le mouvement est inversé pour la problématique de l'animation (dès 1993 dans les publications francophones, alors qu'elle ne semble émerger qu'en 1999 pour les anglophones). On peut donc bien parler «d'autonomie » de chaque communauté, mais pas de «suivisme ». Le développement a bien été le point de départ de la recherche dans la discipline (mais plutôt conception dans les travaux francophones, et nettement gestion de projets pour les anglophones). Enfin, l'évaluation, problématique dominante de la discipline, a été moins importante globalement dans les recherches francophones (20\% contre $27 \%$ ), mais plus constante.

Cet intérêt plus ancien des francophones pour les questions d'animation doit être sans aucun doute relié à la perspective de recherche adoptée (tableau 5) : le tournant de l'ingénierie ex-ante vers l'intégration ex-post semble avoir eu lieu dans les années 1993, alors que ce virage n'a été pris qu'en 1999 pour les anglophones.

En croisant les années avec le niveau d'analyse, il est intéressant de noter l'augmentation récente du niveau interorganisationnel (réseaux, EDI, etc.) dans les recherches francophones, et l'augmentation récente du niveau du groupe dans les travaux anglophones à partir de 1999 (on peut sans doute y deviner un engouement pour les études sur les collecticiels). 


\begin{tabular}{|l|c|c|c|}
\hline \multicolumn{4}{|c|}{ Tableau 5 } \\
\hline PERSPECTIVES DANS L'ÉCHANTILLON ANGLOPHONE \\
\hline Années & AV & AP & Total \\
\hline $1977-1980$ & +12 & -8 & $\mathbf{7 2}$ \\
\hline $1981-1983$ & -1 & +1 & $\mathbf{8 1}$ \\
\hline $1984-1985$ & +0 & +0 & $\mathbf{8 0}$ \\
\hline $1987-1989$ & +4 & -3 & $\mathbf{1 1 0}$ \\
\hline $1990-1992$ & +4 & -3 & $\mathbf{9 3}$ \\
\hline $1993-1995$ & -2 & +1 & $\mathbf{8 1}$ \\
\hline $1996-1999$ & -1 & +1 & $\mathbf{6 7}$ \\
\hline $1999-2000$ & -35 & +24 & $\mathbf{5 2}$ \\
\hline Total & $\mathbf{2 6 8}$ & $\mathbf{3 6 8}$ & $\mathbf{6 2 6}$ \\
\hline
\end{tabular}

Note : AV : ingénierie, AP : intégration.

\section{Une évolution dans la façon de mener les recherches : les} anglophones commencent à se tourner vers des démarches interprétatives

Pour l'orientation épistémologique, nous avons déjà noté le fait marquant que les recherches francophones ont toujours utilisé les démarches interprétatives (plus de $30 \%$ de leurs travaux), alors que les travaux anglophones semblent porter un intérêt très récent (depuis 1999) à ce type d'épistémologie, qui ne représente encore que $5 \%$ de leurs recherches.

Dans les publications francophones au contraire, le tournant vers des recherches empiriques plutôt positivistes à partir de 1996 (au détriment des travaux théoriques et surtout normatifs) est sans doute expliqué par l'arrivée de la revue SIM, dont la politique éditoriale était clairement favorable aux études de terrain.
On notera par ailleurs que ces variations en termes d'épistémologie ne se traduisent par aucune évolution historique significative des méthodologies: en croisant les méthodologies avec les périodes, les chi2 ne sont pas significatifs, que ce soit dans les écrits anglophones ou francophones.

\section{CONCLUSION}

Nous avons pu montrer, au vu des sept dimensions étudiées, qu'il existait une différence notable entre les recherches francophones et anglophones. De façon globale, il semble que les travaux anglophones adoptent plus souvent une perspective d'ingénierie, s'intéressant autant que les publications francophones à l'organisationnel mais étant un peu plus attirés par des objets techniques. Ils conduisent de préférence des recherches de type quantitatif avec une épistémologie positiviste. Les travaux fran- 
cophones, au contraire, ont plutôt tendance à s'orienter vers des thèmes liés à l'animation des SI, dans une perspective ex-post, délaissant la technique au profit du conceptuel, avec un recours plus important aux méthodologies qualitatives et à l'épistémologie interprétative.

Nous avons pu montrer ensuite qu'il existait une évolution différente dans les deux groupes. On peut bien parler « d'autonomie » de chaque communauté, mais pas de « suivisme » systématique. Les écrits anglophones précèdent les francophones dans l'intérêt porté à la problématique de la gestion stratégique et aux démarches positivistes, mais les écrits francophones précèdent les anglophones dans l'intérêt porté à la problématique de l'animation des SI et aux démarches interprétatives.

Il est intéressant, face à cette différence d'approche des thèmes et des perspectives, de revenir sur l'identité de la discipline « SI ».

La discipline SI est récente, c'est la dernière née des branches des « sciences de gestion ». L'association AIM (Association information et management) est la plus jeune des associations nationales de sciences de gestion, et la revue SIM, née sous l'impulsion de Frantz Rowe et Robert Reix, première revue française en SI, n'a vu le jour qu'en 1996.

Cette grande jeunesse explique les questionnements récurrents sur l'identité de la discipline. Régulièrement les auteurs en SI s'interrogent sur ce qui fonde leur discipline.

De façon pragmatique, on peut définir la discipline de plusieurs façons:

- en étudiant le contenu des manuels « SI » de référence,
- en étudiant les thèses soutenues dans la discipline,

- en étudiant les articles publiés par des revues scientifiques de la discipline.

Quel que soit l'axe d'analyse privilégié, force est de constater, au vu du fonctionnement du système académique, qu'une problématique en SI devient telle lorsqu'elle est cautionnée par un acteur académique exerçant du pouvoir dans la discipline. Une thèse en SI le devient lorsqu' un acteur habilité à diriger des recherches en décide ainsi, et lorsque deux rapporteurs partagent cette opinion. La logique est la même en ce qui concerne le fonctionnement des revues scientifiques: un article sera publié comme un article en SI lorsque deux personnes du comité de programme de la revue acceptent l'idée qu'il en soit ainsi.

Ainsi, si les problématiques de SI sont évolutives, et l'histoire de la discipline le montre, elles le sont en fonction de l'évolution des acteurs la constituant. Il est intéressant de noter à cet égard, que les « pères fondateurs » de la discipline ont un profil différent des acteurs actuels. En effet, les pionniers des SI ont reçu une formation soit en ingénierie, soit en sciences économiques et sociales. Ces pères ont influencé la recherche en fonction de leurs propres origines. On voit seulement émerger depuis peu de temps aux postes à responsabilités des acteurs ayant reçu une formation d'origine en SI, et cela influencera l'avenir de la discipline. Dans un premier temps, on peut imaginer que la question identitaire (« qui sommes-nous »?) soit moins prégnante chez ces derniers que chez les pères fondateurs. En effet les acteurs issus d'une formation en SI identifient certainement plus clairement les fron- 
tières de la discipline que les anciens, justement grâce au travail de pionnier de ces anciens. On peut donc supposer que les jeunes chercheurs en SI n'aient aucun complexe à développer l'axe intitulé «Autres » dans notre grille de lecture. C'est un principe de systémique qui est mis ici en évidence : un système, pour survivre, a besoin d'un certain degré d'ouverture avec son environnement, afin de pouvoir évoluer. Sans ouverture, la sclérose et la mort menacent. D'un autre côté, une trop grande ouverture est dangereuse dans le sens où la dilution dans l'environnement menace: c'est le problème de l'identité. Notre pari est que la discipline SI est suffisamment constituée de part un fonctionnement performant du système académique pour ne plus avoir à se poser le problème de l'ouverture sur l'environnement. Les chercheurs peuvent alors sans complexe faire preuve de créativité et d'ouverture dans la définition des problématiques auxquelles ils s'intéressent et apporter sans cesse un renouveau à une discipline par nature totalement évolutive.

\section{BibLIOGRAPHIES}

Alavi M., Carlson P., “A Review of MIS Research and Disciplinary Development”, Journal of Management Information Systems, Spring, vol. 8, $\mathrm{n}^{\circ}$ 4, 1992, p. 45-62.

Barki H., Rivard S., Talbot J., “An Information Systems Keywords Classification Scheme”, MIS Quaterly, vol. 12, n², June 1988, p. 298-322.

Barki H., Rivard, S., Talbot J., "A Classification Scheme for IS Research Literature: an Update”, MIS Quaterly, vol. 17, n², June 1993, p. 209-226.

Brancheau J.C., Janz B.D., Wetherbe J.C., "Key Issues in Information Systems Management: 1994-95 SIM Delphi results”, MIS Quaterly, vol. 20, n² 2, June 1996, p. 225-242.

Cheon M.J., Lee C.C, Grover V., "Research in MIS - Points of Work and Reference: a Replication and Extension of the Culnan and Swanson Study", Database, Spring 1991, p. 21-29.

Cheon M.J., Grover V., Sabherwal R., "The Evolution of Empirical Research in IS" Information and Management, vol. 24, 1993, p. 107-119.

Claver E., Gonzalez R., Liopis J., “An Analysis of Research in Information Systems (19811997)", Information and Management, vol. 37, 2000, p. 181-195.

Culnan M.J., Swanson E.B., "Research in Management Information Systems, 1980-1984: Points of Work and Reference", MIS Quaterly, vol. 10, n³ 3, September 1986, p. 289-301.

Culnan M.J., "Mapping the Intellectual Structure of MIS, 1980-1985: a Co-citation Analysis", MIS Quaterly, vol. 11, n³, September 1987, p. 341-353.

Desq S., Fallery B., Reix R., Rodhain F., “25 ans de recherches en Systèmes d'Information”, Systèmes d'Information et Management, vol. 7, $\mathrm{n}^{\circ}$ 3, 2002, p. 5-31.

Dickson G.W., Leitheiser R.L., Wetherbe J.C., Nechis M., "Key Information Systems Issues for the 1980's", MIS Quaterly, vol. 8, September 1984, p. 135-146. 
Gillenson M.L., Stutz J.D., “Academic Issues in MIS: Journals and Books”, MIS Quaterly, vol. 15, December, 1991, p. 447-452.

Hamilton S., Ives B., "Knowledge Utilization among MIS Researchers", MIS Quaterly, vol. 6, December 1982, p. 61-77.

Hamilton S., Ives B., "MIS Research Strategies", Information and Management, vol. 5, $\mathrm{n}^{\circ} 6$, 1982, p. 339-347.

Ives B., Hamilton S., Davis G.B., “A Framework for Research in Computer-based Management Information Systems”, Management Science, vol. 26, n 9, September 1980, p. 910-934.

Lai V.S., Mahapatra R.K., "Exploring the Research in Information Technology Implementation", Information and Management, vol. 32, 1997, p. 187-201.

Lee A., “Inaugural Editor's Comments”, MIS Quarterly, vol. 23, n 1, March 1999.

Lee Z., Gosain S, Im I., “ Topics of Interest in IS : Evolution of Themes and Differences between Research and Practice ”, Information and Management, vol. 36, 1999, p. 233-246.

Peaucelle J.L., «La recherche française en systèmes d'information, comparaison avec les États-Unis », Systèmes d'Information et Management, vol. 6, n 3, 2001, p. 5-30.

Reix R., Fallery B., «Systèmes d'information: problématiques et paradigmes », Communication aux Journées de la Recherche FNEGE, Paris, Octobre 1996.

Suomi R., "On the Nationality Balance of Authors and References in Selected MIS Journals", Information and Management, vol. 24, 1993, p. 339-347.

Swanson E. B., Ramiller N.C., "Information Systems Research Thematics: Submissions to a New Journal , 1987-1992”, Information Systems Research, vol. 4, n 4, 1993, p. 299-330. 


\section{DESCRIPTIF DES 13 PROBLÉMATIQUES DÉFINIES A PRIORI POUR CLASSER LES RECHERCHES EN SI}

1. Gestion stratégique (par exemple: Jean-Luc Lapon, Paris I, thèse de doctorat: «La contribution de la direction informatique à l'alignement stratégique : une analyse des types de directions alignées dans les grandes entreprises françaises »)

1.1 Utilisation stratégique des systèmes d'information, systèmes d'information à avantage concurrentiel, veille stratégique, veille technologique

1.2 Planification des systèmes d'information, méthodes de planification, schéma directeur 1.3 Externalisation, infogérance, outsourcing, gestion des investissements en technologies de l'information

1.4 Architecture technologique, centralisation/ décentralisation, informatique distribuée, client-serveur, organisation des moyens techniques, systèmes ouverts, administration générale des données, intégration des systèmes d'information

1.5 Économie de l'information et des TI, marché des TI, niveau macro-économique, diffusion des TI et des innovations, comparaisons internationales, différences interculturelles, réglementation, droit

2. Développement (par exemple: Jérome Steffe, thèse de doctorat: «Contribution à la modélisation du système d'information de l'exploitation agricole »)

2.1 Définition des besoins en information, analyse de l'existant, diagnostic, méthodes de conception, modélisation, modèles de données, modèles de traitements, méthodes objet

2.2 Outils de développement, langages de $4^{\mathrm{e}}$ génération, programmation, Atelier de Génie Logiciel ou Computer Aided Software Engineering, réutilisation

2.3 Approches de développement, gestion des projets d'informatisation. Cycle de développement, prototypage, Rapid Application Design, évaluation des risques du projet, estimation des charges de développement, méthodes de gestion de projet

2.4 Implantation, formation des utilisateurs, test des programmes, réception, conversion

2.5 Maintenance, entretien des systèmes d'information

3. Contrôle (par exemple: Hajer Kefi, thèse de doctorat: «Évaluation des technologies et systèmes d'information: cas d'un entrepôt de données implanté dans une institution financière »)

3.1 Conduite du changement organisationnel, reengineering des processus, conséquences organisationnelles, appropriation des technologies, promotion de la diffusion

3.2 Evaluation, mesures d'utilisation, de satisfaction, de performances, qualité des logiciels, audit informatique, sécurité informatique

3.3 Gestion des personnels informaticiens, compétences, organisation de la fonction

4. Autres (par exemple « éthique et systèmes d'information »). 


\section{LA DESCRIPTION DES SEPT GRANDES DIMENSIONS SUR L'ENSEMBLE DE L'ÉCHANTILLON}

\begin{tabular}{|l|c|c|c|}
\hline Problématiques $^{1}$ & Problématiques initiales & Nb. citations & Fréquence \\
\hline Gestion stratégique & $1.1,1.2,1.3,1.4$ & 167 & $16 \%$ \\
\hline Économie et divers & $1.5,3.3,4$ & 160 & $16 \%$ \\
\hline Total Stratégie & & & $\mathbf{3 2} \%$ \\
\hline Conception & 2.1 & 114 & $11 \%$ \\
\hline Gestion projet & $2.2,2.3,2.4,2.5$ & 172 & $17 \%$ \\
\hline Total Développement & & & $\mathbf{2 8} \%$ \\
\hline Animation & 3.1 & 152 & $15 \%$ \\
\hline Evaluation & 3.2 & 252 & $25 \%$ \\
\hline Total Contrôle & & & $\mathbf{4 0} \%$ \\
\hline
\end{tabular}

\begin{tabular}{|l|c|c|}
\hline Domaine & Nb. citations & Fréquence \\
\hline Informationnel & 84 & $8 \%$ \\
\hline Fonctionnel & 73 & $7 \%$ \\
\hline Décisionnel & 142 & $14 \%$ \\
\hline Relationnel & 124 & $12 \%$ \\
\hline Général & 340 & $33 \%$ \\
\hline
\end{tabular}

\begin{tabular}{|l|c|c|}
\hline Perspective & Nb. citations & Fréquence \\
\hline $\begin{array}{l}\text { Ex-ante } \\
\text { (ingénierie) }\end{array}$ & 363 & $36 \%$ \\
\hline $\begin{array}{l}\text { Ex-post } \\
\text { (intégration) }\end{array}$ & 611 & $60 \%$ \\
\hline
\end{tabular}

\begin{tabular}{|l|c|c|}
\hline \multicolumn{1}{|c|}{ Objet } & Nb. citations & Fréquence \\
\hline Technique & 74 & $7 \%$ \\
\hline Organisationnel & 860 & $84 \%$ \\
\hline Conceptuel & 81 & $8 \%$ \\
\hline
\end{tabular}

\begin{tabular}{|l|c|c|}
\hline \multicolumn{1}{|c|}{ Épistélologie } & Nb. citations & Fréquence \\
\hline Positiviste & 454 & $45 \%$ \\
\hline Interprétatif & 138 & $14 \%$ \\
\hline $\begin{array}{l}\text { Théorique, } \\
\text { normatif }\end{array}$ & 335 & $33 \%$ \\
\hline
\end{tabular}

\begin{tabular}{|l|c|c|}
\hline Niveau d'analyse & Nb. citations & Fréquence \\
\hline Individuel & 283 & $28 \%$ \\
\hline Groupe & 51 & $5 \%$ \\
\hline Organisationnel & 502 & $49 \%$ \\
\hline Interorganisationnel & 51 & $5 \%$ \\
\hline Société & 47 & $5 \%$ \\
\hline
\end{tabular}

\begin{tabular}{|l|c|c|}
\hline Méthodologie & Nb. Citations & Fréquence \\
\hline Conceptuel & 352 & $35 \%$ \\
\hline Quantitatif & 233 & $23 \%$ \\
\hline Qualitatif & 333 & $33 \%$ \\
\hline Expérimental & 101 & $10 \%$ \\
\hline
\end{tabular}

1. Les 13 problématiques initiales ont été regroupées en 6 problématiques plus larges après codage des articles. 
